\title{
FINITE GENERALIZED TRIANGLE GROUPS
}

\author{
J. HOWIE, V. METAFTSIS AND R. M. THOMAS
}

\begin{abstract}
We give an almost complete classification of those generalized triangle groups that are finite, building on previous results of Baumslag, Morgan and Shalen [1], Conder [4], Rosenberger [12] and Levin and Rosenberger [11]. There are precisely two groups for which we cannot decide whether or not they are finite.
\end{abstract}

\section{INTRODUCTION}

A generalized triangle group is one given by a presentation

$$
\left\langle a, b \mid a^{p}, b^{q}, W^{r}\right\rangle,
$$

where $p, q, r$ are integers greater than 1 , and $W$ is a word of the form

$$
a^{\alpha_{1}} b^{\beta_{1}} \ldots a^{\alpha_{k}} b^{\beta_{k}}
$$

$k \geq 1,0<\alpha_{i}<p, 0<\beta_{i}<q$ for all $i$, which is not a proper power.

These groups are a natural generalization of the triangle groups $T=T(p, q, r)$ defined by the presentations

$$
\left\langle a, b \mid a^{p}, b^{q},(a b)^{r}\right\rangle,
$$

where (again) $p, q$ and $r$ are integers greater than one. It is known that, in $T$, the elements $a, b$ and $a b$ really do have orders $p, q$ and $r$ respectively (i.e., the group does not "collapse"), and that $T$ is infinite if $\frac{1}{p}+\frac{1}{q}+\frac{1}{r} \leq 1$ and finite if $\frac{1}{p}+\frac{1}{q}+\frac{1}{r}>1$.

The first two of these properties go over to generalized triangle groups. It was shown in [1], and, independently, in [6], that, if $G$ is the generalized triangle group defined by

$$
\left\langle a, b \mid a^{p}, b^{q}, W^{r}\right\rangle,
$$

then there is a homomorphism $\phi: G \rightarrow P S L(2, \mathbb{C})$ such that $\phi(a), \phi(b)$ and $\phi(W)$ have orders $p, q$ and $r$ respectively. Around the same time Boyer in [2] showed that there is a homomorphism $G \rightarrow \mathrm{SO}(3)$ with the same property. He also showed that $G$ is infinite if no two of $p, q, r$ are equal to 2 and $\max \{p, q, r\} \geq 6$, under some restrictions on $W$.

It was shown in [8] that $G$ is infinite if $\frac{1}{p}+\frac{1}{q}+\frac{1}{r} \leq 1$ provided that $r \geq 3$, or $r=2$ with $p \geq 3$ and $q \geq 6$, or $(p, q, r)=(4,5,2)$. This was generalized

Received by the editors July 9, 1993 and, in revised form, October 31, 1994.

1991 Mathematics Subject Classification. Primary 20F05.

The third author would like to thank Hilary Craig for all her help and encouragement. 
in [1], where it was shown that $G$ is infinite whenever $\frac{1}{p}+\frac{1}{q}+\frac{1}{r} \leq 1$. An elementary proof of this last fact (given that $G$ does not collapse) may be found in [13].

However, unlike the triangle groups, a generalized triangle group may be infinite when $\frac{1}{p}+\frac{1}{q}+\frac{1}{r}>1$. It is an open question as to precisely which generalized triangle groups are finite, and it is this question that we address in this paper. The finite generalized groups with presentation

$$
\left\langle a, b \mid a^{p}, b^{q},\left(a^{\alpha_{1}} b^{\beta_{1}} \ldots a^{\alpha_{k}} b^{\beta_{k}}\right)^{r}\right\rangle,
$$

have been determined for $r \geq 3$ in [7]. For $k=1$ the group is finite only if it is a triangle group (see for example [3]). The finite cases have also been determined for $r=2, k=2$ in [12], for $r=2, k=3$ or 4 in [11], and, if $(p, q)=(2,3)$, for $k r \leq 12$ in [4]. Building on these results, we prove the following:

Theorem 1.1. Let $G=\left\langle a, b \mid a^{p}, b^{q}, W^{r}\right\rangle$ be a finite generalized triangle group, where $W=a^{\alpha_{1}} b^{\beta_{1}} \ldots a^{\alpha_{k}} b^{\beta_{k}}, 0<\alpha_{i}<p, 0<\beta_{i}<q, W$ is not a proper power, and $k \geq 2$. Then up to equivalence $G$ is one of the following:

(1) $\left\langle a, b \mid a^{2}, b^{3},\left(a b a b a b^{2} a b^{2}\right)^{2}\right\rangle$, of order 576 ;

(2) $\left\langle a, b \mid a^{2}, b^{3},\left(a b a b a b^{2}\right)^{3}\right\rangle$, of order 1440 ;

(3) $\left\langle a, b \mid a^{3}, b^{3},\left(a b a b^{2}\right)^{2}\right\rangle \cong A_{5} \times C_{3}$, of order 180 ;

(4) $\left\langle a, b \mid a^{3}, b^{3},\left(a b a^{2} b^{2}\right)^{2}\right\rangle$, of order 288;

(5) $\left\langle a, b \mid a^{2}, b^{5},\left(a b a b^{2}\right)^{2}\right\rangle$, of order 120 ;

(6) $\left\langle a, b \mid a^{2}, b^{5},\left(a b a b a b^{4}\right)^{2}\right\rangle$, of order 1200 ;

(7) $\left\langle a, b \mid a^{2}, b^{5},\left(a b a b^{2} a b^{4}\right)^{2}\right\rangle$, of order 1200;

(8) $\left\langle a, b \mid a^{2}, b^{4},\left(a b a b a b^{3}\right)^{2}\right\rangle$, of order 192;

(9) $\left\langle a, b \mid a^{2}, b^{3},\left(a b a b^{2}\right)^{2}\right\rangle$, of order 24 ;

(10) $\left\langle a, b \mid a^{2}, b^{3},\left(a b a b a b^{2}\right)^{2}\right\rangle$, of order 48 ;

(11) $\left\langle a, b \mid a^{2}, b^{3},\left(a b a b a b a b^{2}\right)^{2}\right\rangle$, of order 120 ;

(12) $\left\langle a, b \mid a^{2}, b^{3},\left(a b a b a b^{2} a b a b^{2}\right)^{2}\right\rangle$, of order 720 ;

(13) $\left\langle a, b \mid a^{2}, b^{3},\left(a b a b a b a b a b^{2} a b^{2}\right)^{2}\right\rangle$, of order 2880 ; or possibly

(14) $\left\langle a, b \mid a^{2}, b^{3},\left(a b a b a b a b^{2} a b a b^{2} a b^{2}\right)^{2}\right\rangle$, or

(15) $\left\langle a, b \mid a^{2}, b^{3},\left(a b a b a b a b^{2} a b^{2} a b a b^{2} a b^{2}\right)^{2}\right\rangle$.

By the term "equivalence" here we mean the following. We regard two cyclically reduced words $W, W^{\prime} \in \mathbb{Z}_{p} * \mathbb{Z}_{q}$ as equivalent if we can transform one to the other by a sequence of moves of the form:

(1) cyclic permutation;

(2) inversion;

(3) automorphism of $\mathbb{Z}_{p}$ or of $\mathbb{Z}_{q}$; or

(4) interchanging the two free factors (if $p=q$ ).

By abuse of language, if two words are equivalent, we say also that the corresponding generalized triangle groups are equivalent. It is clear that equivalent groups are isomorphic, so it suffices to study generalized triangle groups up to equivalence.

In this setting, Theorem 1.1 is almost a complete classification of finite generalized triangle groups. We do not know whether either or both of the last two groups listed in the theorem are finite. We do know, however, that they 
are fairly large. They have homomorphic images of orders $453,600,000$ and $17,694,720$ respectively.

The two groups listed of order 1200 are isomorphic, since $\left(a b a b a b^{4}\right)^{2}$ and $\left(a b a b^{2} a b^{4}\right)^{2}$ have the same normal closure in $\left\langle a, b \mid a^{2}, b^{5}\right\rangle$. The two groups of order 120 are also isomorphic-both are isomorphic to $C_{2} \times A_{5}$. Also, none of the above are isomorphic to finite triangle groups (the only group listed that has the same order as a finite triangle group is isomorphic to $C_{2} \times A_{4}$ rather than to $S_{4}$ ). Hence up to isomorphism there are between 11 and 13 finite generalized triangle groups that are not triangle groups.

The study of generalized triangle groups is of interest for a number of reasons. For instance, they provide accessible examples of one-relator products (see for example [5] for details). In particular, finite generalized triangle groups that are not themselves triangle groups yield examples of one-relator products that do not behave in quite the expected way with respect to group cohomology for example. See [5], in particular Theorem 4.1.

One interesting question not addressed here is the following. The ordinary triangle groups satisfy a so-called Tits alternative-either the group has a soluble subgroup of finite index, or it contains a nonabelian free subgroup. Rosenberger [12] has conjectured that a Tits alternative also holds for generalized triangle groups, and there is a lot of evidence in favour of that conjecture $[1,7,11,12]$.

The methods we use are mainly those of $[1,6,11,12]$, namely analysis of representations of generalized triangle groups, particularly using trace polynomials. We elaborate on this in Section 2 below. For part of the proof of Theorem 1.1 we also require the technique of pictures as in $[5,9,10]$. We recall the basic definitions of pictures in Section 3.

The proof of the main theorem is spread over Sections 4, 5 and 6, which treat separately the various possible values of the triple $(p, q, r)$. The various parts of the proof are then collected together in Section 7.

\section{REPRESENTATIONS AND TRACE POLYNOMIALS}

For any group $G$, let $Z(G)$ denote the centre of $G$. If $R$ is any commutative ring with unity, $G L(2, R)$ denotes the group of $2 \times 2$ invertible matrices with elements from $R, S L(2, R)$ denotes the elements of $G L(2, R)$ with determinant 1 , and $P S L(2, R)$ denotes the factor group $S L(2, R) / Z(S L(2, R))$.

Now let us restrict to the case where $R=\mathbb{C}$. Let $I$ be the identity matrix and $\operatorname{tr}(A)$ the trace of matrix $A$. If $A \in S L(2, \mathbb{C})$ then it is well known that $A$ has finite order $m>2$ if and only if $\operatorname{tr}(A)=\alpha+\alpha^{-1}$ for some primitive $m$ th root $\alpha$ of unity in $\mathbb{C}$. Equivalently $\operatorname{tr}(A)=2 \cos \frac{2 s \pi}{m}$ with $s \in \mathbb{N}$ and $(m, s)=1$. It is also easy to show (by direct calculation) that if $A, B \in S L(2, \mathbb{C})$, then $\operatorname{tr}(A B)+\operatorname{tr}\left(A^{-1} B\right)=\operatorname{tr}(A) \operatorname{tr}(B)=\operatorname{tr}(A B)+\operatorname{tr}\left(A B^{-1}\right)$.

If $A \in S L(2, \mathbb{C})$ is an element of order 2 , then $A=-I$, and also, if $\operatorname{tr}(A)=0$, then $A^{2}=-I$.

If $G=\left\langle a, b \mid a^{p}, b^{q}, W^{r}\right\rangle$ is a generalized triangle group, then, following [1], we say that a homomorphism $\rho: G \rightarrow H$ is a special representation if $\rho(a)$, $\rho(b), \rho(W)$ have orders $p, q, r$ respectively. Usually we consider special representations to matrix groups. Three key results here are as follows.

Theorem 2.1. ([1], see also [6]) Every generalized triangle group admits a special representation to $\operatorname{PSL}(2, \mathbb{C})$. Moreover, this representation can be chosen to 
send the generators $a, b$ to conjugates of any chosen elements of orders $p, q$ respectively.

Theorem 2.2. ([1]) If a generalized triangle group $G$ has a special representation to a cyclic group, then $G$ is infinite.

Theorem 2.3. The only finite subgroups of $\operatorname{PSL}(2, \mathbb{C})$ are the cyclic groups $C_{n}$ $(n \geq 1)$, the dihedral groups $D_{n}(n \geq 2)$, the alternating groups $A_{4}$ and $A_{5}$ and the symmetric group $S_{4}$.

For any representation $\rho:\left\langle a, b \mid a^{2 p}, b^{2 q}\right\rangle \rightarrow S L(2, \mathbb{C})$ we have that the trace $\operatorname{tr} \rho(W)$ of $\rho(W)$ is given by a polynomial in the traces of $\rho(a), \rho(b)$, $\rho(a b)$ :

$$
f_{W}(\operatorname{tr} \rho(a), \operatorname{tr} \rho(b), \operatorname{tr} \rho(a b)) .
$$

Moreover the degree of the polynomial $f$ in the third variable is at most $k$, that is, half the (free product) length of $W$. (See Lemma 1 in [1].) Actually, if the first two variables of $f$ are fixed as $2 \cos (\pi / p)$ and $2 \cos (\pi / q)$ respectively, then an easy inductive argument shows that $f$ becomes a polynomial of degree precisely $k$ in the single variable $\operatorname{tr}(\rho(a b))$. We refer to this as the trace polynomial of $W$, and write it as $\tau_{W}$. Note that the values of $p, q$ are involved implicitly in the definition of $\tau_{W}$, but we are suppressing them in the notation. This should cause no confusion in what follows.

Lemma 2.4. Let $U \in S L(2, \mathbb{C}[X])$ be a matrix and suppose that $\alpha \in \mathbb{C}$ is a root of $\operatorname{tr}(U)$ of multiplicity $m>0$. Then $U$ has infinite order in the group $\operatorname{PSL}\left(2, \mathbb{C}[X] /\left((X-\alpha)^{m+1}\right)\right)$.

Proof. Let $\operatorname{tr}(U)=(X-\alpha)^{m} q(X)$. Then $q(\alpha) \neq 0$ and $U^{2}=V-I$, where $V=$ $(X-\alpha)^{m} q(X) U$. Notice that the entries of $U$ are not all multiples of $(X-\alpha)$, since $\operatorname{det}(U)=1$. Hence $V \neq 0$ as a matrix over $\Lambda=\mathbb{C}[X] /\left((X-\alpha)^{m+1}\right)$. However $V^{2}=0$ as a matrix over $\Lambda$. Hence $U^{2 k}=(-1)^{k} I+(-1)^{k-1} k V \neq \pm I$ in $S L(2, \Lambda)$ for all positive integers $k$. Hence $U^{2}$, and so also $U$, has infinite order in $P S L(2, \Lambda)$, as claimed.

Now fix integers $p, q, r \geq 2$. Suppose $A, B \in S L(2, \mathbb{C})$ are matrices with $\operatorname{tr}(A)=2 \cos (\pi / p)$ and $\operatorname{tr}(\bar{B})=2 \cos (\pi / q)$. Let $W$ be a cyclically reduced word of (free product) length at least 2 in $\left\langle a \mid a^{p}\right\rangle *\left\langle b \mid b^{q}\right\rangle$, that is not a proper power, and let $\tau_{W}=\tau_{W}(X)$ be the trace polynomial of $W$. Define a polynomial

$$
\sigma(X)=\prod_{\alpha}\left(\tau_{W}(X)-\alpha-\alpha^{-1}\right),
$$

where the product is taken over all primitive $r$ th roots $\alpha$ of -1 if $r$ is even and of \pm 1 if $r$ is odd, with nonnegative imaginary part. Thus, if $r=2,3,4$ or 5, then $\sigma(X)$ is, respectively, $\tau_{W}(X), \tau_{W}(X)^{2}-1, \tau_{W}(X)^{2}-2$ or $\tau_{W}(X)^{4}-$ $3 \tau_{W}(X)^{2}+1$. Then $\sigma(\operatorname{tr}(A B))=0$ if and only if $\operatorname{tr}(W(A, B))= \pm\left(\alpha+\alpha^{-1}\right)$ for $\alpha$ as above, which is the case if and only if $W(A, B)$ has order $r$ in $\operatorname{PSL}(2, \mathbb{C})$.

Theorem 2.5. If $\sigma(X)$, as defined above, has a multiple root, then the generalized triangle group $G=\left\langle a, b \mid a^{p}, b^{q}, W^{r}\right\rangle$ is infinite.

Proof. Suppose $\beta$ is a multiple root of $\sigma(X)$. By Theorem 2.1 we can find a special representation $\rho_{1}: G \rightarrow P S L(2, \mathbb{C})$ such that $\operatorname{tr}\left(\rho_{1}(a b)\right)=\beta$, and 
the image of $\rho_{1}$ is infinite unless $\beta$ has the form $\alpha+\alpha^{-1}$ for some $2 m$ th root of unity $\alpha \neq \pm 1$. Moreover, if the image of $\rho_{1}$ is finite and $\rho_{1}(a b)$ has order greater than 6 , then $\rho_{1}$ must be cyclic (it cannot be dihedral, since $(p, q) \neq(2,2))$, so $G$ is infinite by Theorem 2.2. Assume then that $\beta$ does indeed have the form $\alpha+\alpha^{-1}$ for some $2 m$ th root of unity $\alpha \neq \pm 1$, with $m \leq 5$. Choose integers $k$ coprime to $p$ and $\ell$ coprime to $q$ such that $(\cos (k \pi / p)+\cos (\ell \pi / q))^{2} \neq \beta+2$. (It is clear that this can be done provided $\phi(p) \phi(q)>2$, where $\phi$ is the Euler function, while if $p=2$ and $q \in\{3,4,6\}$ the inequality is automatically true.)

Now define matrices $A, B, C \in S L(2, \mathbb{C}[X])$ by

$$
A=\left(\begin{array}{cc}
0 & 1 \\
-1 & 2 \cos (k \pi / p)
\end{array}\right), \quad B=\left(\begin{array}{cc}
0 & 1 \\
-1 & 2 \cos (\ell \pi / q)
\end{array}\right), \quad C=\left(\begin{array}{cc}
1 & X \\
0 & 1
\end{array}\right) .
$$

Then define $f(X)=\operatorname{tr}\left(A C B C^{-1}\right)=(-X+2 \cos (\pi / p))(X+2 \cos (\pi / q))-2$. Choose $\gamma \in \mathbb{C}$ such that $f(\gamma)=\beta$, and let $\Lambda=\mathbb{C}[X] /\left((X-\gamma)^{2}\right)$. Since $\beta$ is a multiple root of $\sigma$, it follows that $\gamma$ is a multiple root of $\sigma \circ f$, and hence that there is a representation $\rho_{2}: G \rightarrow P S L(2, \Lambda)$ given by $a \mapsto A$, $b \mapsto C B C^{-1}$. In order to prove that $G$ is infinite, it suffices to find, by Lemma 2.4 , a cyclically reduced free product word $u$ in $a, b$ such that $\gamma$ is a simple root of $\operatorname{tr}\left(\rho_{2}(u)\right)=\tau_{u}(f(X))$. Now the quadratic polynomial $f(X)-\beta$ has distinct roots, since $(\cos (k \pi / p)+\cos (\ell \pi / q))^{2} \neq \beta+2$. Hence $\gamma$ is a simple root of $\operatorname{tr}\left(\rho_{2}(u)\right)$ if and only if $\beta$ is a simple root of $\tau_{u}$.

To complete the proof, we exhibit, for each possible combination of $p, q$ and $\beta$, a suitable word $u$ in $a, b$. We assume that $p \leq q$ and that $1 / p+1 / q>1 / 2$, since $1 / p+1 / q+1 / r>1$ (for otherwise $G$ is automatically infinite, by [1]).

$(p, q)=(2,2)$. $W=a b$, up to conjugacy, and $\sigma$ has no multiple roots.

$(p, q)=(2,3)$. The only noncyclic, finite subgroups of $\operatorname{PSL}(2, \mathbb{C})$ generated by elements of orders 2 and 3 are $S_{3}, A_{4}, S_{4}$ and $A_{5}$, and the only possible orders of elements in these groups are $1,2,3,4$ and 5 . Hence $\beta \in$ $\left\{0, \pm 1, \pm \sqrt{2}, \frac{ \pm 1 \pm \sqrt{5}}{2}\right\}$. Then $a b$ has trace polynomial $X$, while the trace polynomial of $(a b)^{3}\left(a b^{2}\right)^{2} a b\left(a b^{2}\right)^{2}$ has simple roots $\pm 1, \pm \sqrt{2}, \frac{ \pm 1 \pm \sqrt{5}}{2}$.

$(p, q)=(2,4)$. Here the finite subgroups in question are $D_{8}$ and $S_{4}$, with elements of order 1,2,3 and 4. However, in each of these groups, if a generating pair of elements have orders 2 and 4 , then their product has order less than 4. Hence $\beta \in\{0, \pm 1\}$. The word $a b a b a b^{3}$ has trace polynomial with simple roots $0, \pm 1$.

$(p, q)=(2,5)$. The finite subgroups are $D_{10}$ and $A_{5}$, with elements of orders $1,2,3$ and 5 . However, in $A_{5}$, if the product $z$ of elements $x, y$ of orders 2 and 5 respectively also has order 5 , then $z$ is conjugate to $y^{2}$. In terms of traces, this means that if $\operatorname{tr}(y)= \pm 2 \cos (\pi / 5)= \pm \frac{1+\sqrt{5}}{2}$, then $\operatorname{tr}(z)=$ $\pm 2 \cos (2 \pi / 5)= \pm \frac{1-\sqrt{5}}{2}$. Hence we may assume that $\beta \in\left\{0, \pm 1, \pm \frac{1-\sqrt{5}}{2}\right\}$. The trace polynomial of $a b a b a b^{3} a b^{2} a b^{4}$ has all five of these as simple roots.

$(p, q)=(2, n), n>5$. The only noncyclic subgroup of $\operatorname{PSL}(2, \mathbb{C})$ generated by an element of order 2 and one of order $n$ is $D_{2 n}$, in which the product of the two generators has order 2 . Hence we need only consider $\beta=0$, which is the unique simple root of the trace polynomial of $a b$.

$(p, q)=(3,3)$. The only noncyclic finite subgroups of $\operatorname{PSL}(2, \mathbb{C})$ generated by two elements of order 3 are $A_{4}$ and $A_{5}$ with elements of orders 1,2,3 
and 5. Here, if matrices $A, B$ generate $A_{4}$ or $A_{5}$, with $\operatorname{tr}(A)=\operatorname{tr}(B)=1$, then both $A B$ and $A B^{-1}$ have traces in the set $\left\{0, \pm 1, \frac{ \pm 1 \pm \sqrt{5}}{2}\right\}$. In addition, they must satisfy the identity $\operatorname{tr}(A B)+\operatorname{tr}\left(A B^{-1}\right)=\operatorname{tr}(A) \operatorname{tr}(B)=1$, so the set of possible traces is restricted to $\left\{0,1, \frac{1 \pm \sqrt{5}}{2}\right\}$. The trace polynomial of $a b a b^{2} a^{2} b a^{2} b^{2}$ has precisely these four simple roots.

$(p, q)=(3,4)$. The only noncyclic finite subgroup of $\operatorname{PSL}(2, \mathbb{C})$ generated by an element of order 3 and one of order 4 is $S_{4}$, in which case the product of the generators has order either 2 or 4 . As above, we obtain a restriction on the set of traces from the identity $\operatorname{tr}(A B)+\operatorname{tr}\left(A B^{-1}\right)=\operatorname{tr}(A) \operatorname{tr}(B)=\sqrt{2}$. Thus the only traces to be considered are $0, \sqrt{2}$. The trace polynomial of $a b a^{2} b^{2}$ has precisely these two numbers as simple roots.

$(p, q)=(3,5)$. In this case the only finite subgroup of $\operatorname{PSL}(2, \mathbb{C})$ to be considered is $A_{5}$, and the product of the generators has order 2,3 or 5 . The identity $\operatorname{tr}(A B)+\operatorname{tr}\left(A B^{-1}\right)=\operatorname{tr}(A) \operatorname{tr}(B)=\frac{1+\sqrt{5}}{2}$ restricts the possible traces to $0,1, \frac{ \pm 1+\sqrt{5}}{2}$. The trace polynomial of $a b a^{2} b a b^{2} a^{2} b^{4}$ has precisely these values as simple roots.

Thus in all cases we have found a word whose trace polynomial has $\beta$ as a simple root, and so $G$ is infinite, as claimed.

\section{Pictures}

We will give a brief introduction on pictures here. More details can be found in [5], [9] or [10]. Let $G=(A * B) / N\left(r^{m}\right)$ be a one-relator product; then a picture $\Gamma$ on a disc $D^{2}$ over $G$ consists of:

(i) the vertices $u_{1}, \ldots, u_{n}$ (a disjoint union of discs) in the interior of $D^{2}$;

(ii) the $\operatorname{arcs} \xi$ (properly embedded 1 -submanifolds of $D^{2} \backslash \operatorname{int}\left(\bigcup u_{i}\right)$ ) of $\Gamma$;

(iii) a labeling function that associates, to each component of $\partial u_{i} \backslash\{\bigcup \xi\}$ and $\partial D^{2} \backslash\{\bigcup \xi\}$, a label which is an element of $A \cup B$.

The vertex label is the word consisting of all the labels at $u_{i}$, read anticlockwise and is required to be identically equal to $r^{\mp m}$ (up to cyclic permutation).

Every region of $\Gamma$ (that is any component of $D^{2} \backslash \Gamma$ ) either contains labels only from $A$ (an $A$-region) or only from $B$ (a $B$-region). Each edge must separate an $A$-region from a $B$-region. The label of a region is the product of all the labels that appear in its boundary (in the case we are interested in, $A$ and $B$ are abelian, so that this product is well-defined). The label of any region is required to be the identity element of $A$ or of $B$.

Finally, two arcs which together bound a region, are said to be parallel. In particular the two labels in this region are mutually inverse. We also use the term parallel for the equivalence relation generated by this relation. So a class of $k$ parallel arcs identifies 2 subwords of $\left(r^{m}\right)^{ \pm 1}$ of length $k-1$.

A pair of vertices of $\Gamma$ cancel if they are joined by an arc and their vertex labels read from the end points of this arc form mutually inverse words. A picture with no cancelling vertices is a reduced picture.

\section{The CASE $r \geq 3$}

Theorem 4.1. Let $q \geq p \geq 2, r \geq 3$ and let $W$ be a cyclically reduced word of length at least 2 in the free product $\left\langle a \mid a^{p}\right\rangle *\left\langle b \mid b^{q}\right\rangle$, such that $W$ is not $a$ 
proper power. If the generalized triangle group $G=\left\langle a, b \mid a^{p}, b^{q}, W^{r}\right\rangle$ is finite, then either $W$ is equivalent to ab (so $G$ is a triangle group) or $(p, q)=(2,3)$ and $W$ is equivalent to ababab ${ }^{2}$ (in which case $|G|=1440$ ).

Proof. See [7].

\section{THE CASE $q \geq p \geq 3, r=2$}

Theorem 5.1. Let $q \geq p \geq 3$, and let $W$ be a cyclically reduced word of length at least 2 in the free product $\left\langle a \mid a^{p}\right\rangle *\left\langle b \mid b^{q}\right\rangle$ that is not a proper power. If the generalized triangle group $G=\left\langle a, b \mid a^{p}, b^{q}, W^{r}\right\rangle$ is finite, then either $W$ is equivalent to $a b$ (and $G$ is a triangle group), or $p=q=3, r=2$ and $W$ is equivalent to $a b a b^{2}$ or $a b a^{2} b^{2}$ (in which case $G$ has order 180 or 288 respectively).

Proof. We may assume that $1 / p+1 / q+1 / r>1$, so that $p=3, r=2$ and $3 \leq q \leq 5$. For $q=5$ the only finite subgroup of $\operatorname{PSL}(2, \mathbb{C})$ to be considered is $A_{5}$. Assuming $\operatorname{tr}(A)=1$ and $\operatorname{tr}(B)=\frac{1+\sqrt{5}}{2}$, and using the identity $\operatorname{tr}(A B)+\operatorname{tr}\left(A B^{-1}\right)=\operatorname{tr}(A) \operatorname{tr}(B)$, we see that the only possible values for $\operatorname{tr}(A B)$ (and for $\left.\operatorname{tr}\left(A B^{-1}\right)\right)$ are 0,1 and $\frac{ \pm 1+\sqrt{5}}{2}$. For $q=4$ we work in $S_{4}$. If $\operatorname{tr}(A)=1$ and $\operatorname{tr}(B)=\sqrt{2}$ then the only values to be considered for $\operatorname{tr}(A B)$ are $0, \sqrt{2}$. For $q=3$ we must consider $A_{4}$ and $A_{5}$, with $\operatorname{tr}(A)=\operatorname{tr}(B)=1$, so possible values for $\operatorname{tr}(A B)$ are 0,1 and $\frac{1 \pm \sqrt{5}}{2}$.

In all three cases, the maximum degree of $\tau_{W}$ is 4 , so $W$ has length at most 8. The result then follows from [12] and [11].

\section{THE CASE $p=r=2$}

By Sections 4 and 5 we are reduced to consideration of the case where $p=$ $r=2$. If $q=2$ the only possibility is the Klein 4-group; so we assume that $q \geq 3$.

Lemma 6.1. If $q \geq 6$ and $W$ is cyclically reduced of length at least 2 in $\left\langle a \mid a^{2}\right\rangle *\left\langle b \mid b^{q}\right\rangle$, then $G=\left\langle a, b \mid a^{2}, b^{q}, W^{2}\right\rangle$ is finite only if $W$ is $a b^{k}$ or $b^{k}$ a for some $k$ coprime to $q$ (so that $G$ is dihedral).

Proof. The only noncyclic finite subgroups of $\operatorname{PSL}(2, \mathbb{C})$ to contain an element of order $q$ are the dihedral groups, so the only possible value for $\operatorname{tr}(A B)$ is 0 . Hence $\tau_{W}$ has degree 1 , and $W$ has length 2 . In other words $W=a b^{k}$ or $W=b^{k} a$ for some $k$. But if $k$ is not coprime to $q$ then $G$ is a nontrivial free product with amalgamation (and hence infinite).

Theorem 6.2. If $W$ is cyclically reduced of length at least 2 in $\left\langle a \mid a^{2}\right\rangle *\left\langle b \mid b^{5}\right\rangle$, then $G=\left\langle a, b \mid a^{2}, b^{5}, W^{2}\right\rangle$ is finite only if $W$ is equivalent to one of $a b$, $a b a b^{2}, a b a b a b^{4}$ or $a b a b^{2} a b^{4}$ (in which case $G$ has order $10,120,1200$ or 1200 respectively).

Proof. The only noncyclic finite subgroups of $\operatorname{PSL}(2, \mathbb{C})$ that can be generated by an element of order 2 and an element of order 5 are $D_{10}$ and $A_{5}$. If matrices $A, B$ of traces $0, \frac{1+\sqrt{5}}{2}$ respectively generate a subgroup isomorphic to $D_{10}$, then $\operatorname{tr}(A B)=0$, while, if they generate a subgroup isomorphic to $A_{5}$, then 
$\operatorname{tr}(A B)$ is either \pm 1 or $\pm \frac{1-\sqrt{5}}{2}$ (not $\pm \frac{1+\sqrt{5}}{2}$, since $A B$ is not conjugate to $\pm B$ ). Hence $\tau_{W}$ has degree at most 5 , and so $W$ has length at most 10 . If $W$ has length 8 or less, then the result follows from [12] and [11], so we are reduced to the case where $W$ has length 10 .

If $W$ has length 10 and $G$ is finite, then all 5 possible roots occur for $\tau_{W}$. Equivalently, $W$ is mapped to permutations of order 2 under each of the representations: $\quad \rho_{1}: a \mapsto(12)(34), b \mapsto(12345) ; \rho_{2}: a \mapsto(13)(24), b \mapsto$ (12345); and $\rho_{3}: a \mapsto(14)(23), b \mapsto(12345)$. (In fact, the last of these is automatically satisfied, since $\rho_{3}$ is a dihedral representation and $W$ has length 10.) If the exponent sum of $b$ in $W$ is divisible by 5 , then $G$ has a special cyclic representation, and so is infinite by [1]. A computer search reveals that the only words of length 10 mapped to permutations of order 2 by both $\rho_{1}$ and $\rho_{2}$, and having $b$-exponent-sum not divisible by 5 , are, up to equivalence, $W_{k}=a b a b^{3} a b^{2} a b^{4} a b^{k}, 1 \leq k \leq 4$.

Write $V=a b a b^{3} a b^{2} a b^{4} a$, so that $V^{2}=1$ in the free product $\left\langle a \mid a^{2}\right\rangle *$ $\left\langle b \mid b^{5}\right\rangle$, and $W_{k}=V b^{k}$. Then each of the $W_{k}^{2}$ is a consequence of each of the others, together with $a^{2}$ and $b^{5}$. Indeed $W_{4}, W_{3}$ are conjugate to $W_{1}^{-1}, W_{2}^{-1}$ respectively, while $W_{2}^{2}=W_{1}^{2} b^{-1} W_{1}^{2} b$ and similarly $W_{4}^{2}=W_{2}^{2} b^{-2} W_{2}^{2} b^{2}$. Hence there is only one group to be considered, namely

$$
G=\left\langle a, b \mid a^{2}, b^{5}, W_{1}^{2}\right\rangle=\left\langle a, b \mid a^{2}, b^{5}, W_{1}^{2}, W_{2}^{2}\right\rangle .
$$

Consider $G$ as a 2-relator product of $H_{1}=\left\langle a \mid a^{2}\right\rangle$ and $H_{2}=\left\langle b \mid b^{5}\right\rangle$. Suppose that $a b$ has finite order $N$ in $G$, and let $\Gamma$ be a picture on $D^{2}$ over this 2-relator product, with boundary label $(a b)^{N}$. Remove the arcs and regions that meet $\partial D^{2}$ to obtain a tessellated disc (or union of discs, if $\Gamma$ was not connected). Around each vertex labelled $W_{1}^{2}=(V b)^{2}$ or $W_{2}^{2}=\left(V b^{2}\right)^{2}$ we associate angles to the (remaining) corners as follows: every $H_{1}$-cornar has angle 0 , every $H_{2}$-corner labelled by a letter of $V$ has angle $\pi / 3$, while the remaining two $H_{2}$-corners have angle $-\pi / 3$. Thus each interior vertex has angle sum $2 \pi$, and so has zero curvature.

Now suppose there is an interior, positively curved region. We can see that any such region is 2 -sided, with both corners labelled by letters of $V$. Since no letter occurs twice in $V$, we can perform bridge moves on $\Gamma$ until we have a collection of 10 parallel arcs (with label $V$ ) joining a vertex $u$ to a vertex $v$ (see Figure 1). Note that $u \neq v$, since $b^{k} \neq 1$ in $G$.

Now either $u$ and $v$ are a cancelling pair of vertices, or we may replace them by a single new vertex $w$ say (see Figure 2 ). In either case, we replace $\Gamma$ by a smaller picture.

Hence we may assume that there is no positively curved interior region of $\Gamma$. Standard arguments show that there must be a simple boundary vertex of $\Gamma$ (i.e., a boundary vertex that is not a separating vertex) with angle sum less than $\pi$. But this is impossible, since the labels on $\partial D^{2}$ are all $a$ or $b$, so a simple boundary vertex must have at least 5 positive angles (see Figure 3 ).

This contradiction shows that $a b$ has infinite order in $G$, and hence that $G$ is infinite.

Theorem 6.3. If $W$ is cyclically reduced of length at least 2 in $\left\langle a \mid a^{2}\right\rangle *\left\langle b \mid b^{4}\right\rangle$, then $G=\left\langle a, b \mid a^{2}, b^{4}, W^{2}\right\rangle$ is finite only if $W$ is equivalent to one of $a b$, or $a b a b a b^{3}$ (in which case $G$ has order 8 or 192 respectively). 
FINITE GENERALIZED TRIANGLE GROUPS
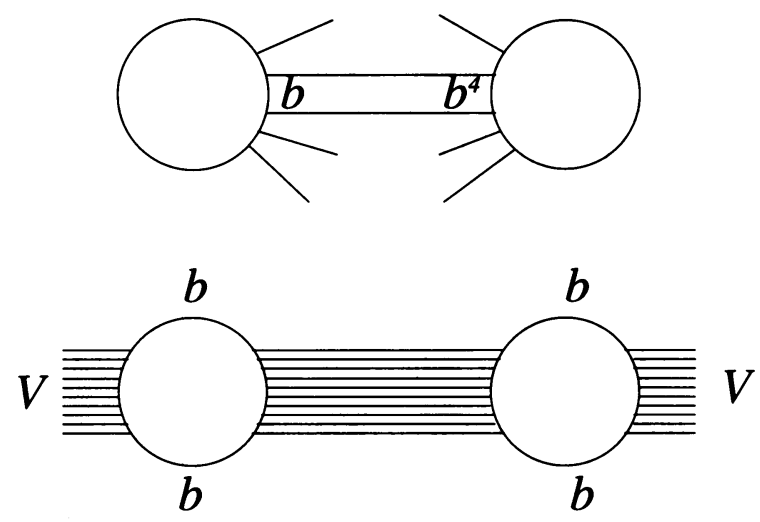

FIGURE 1
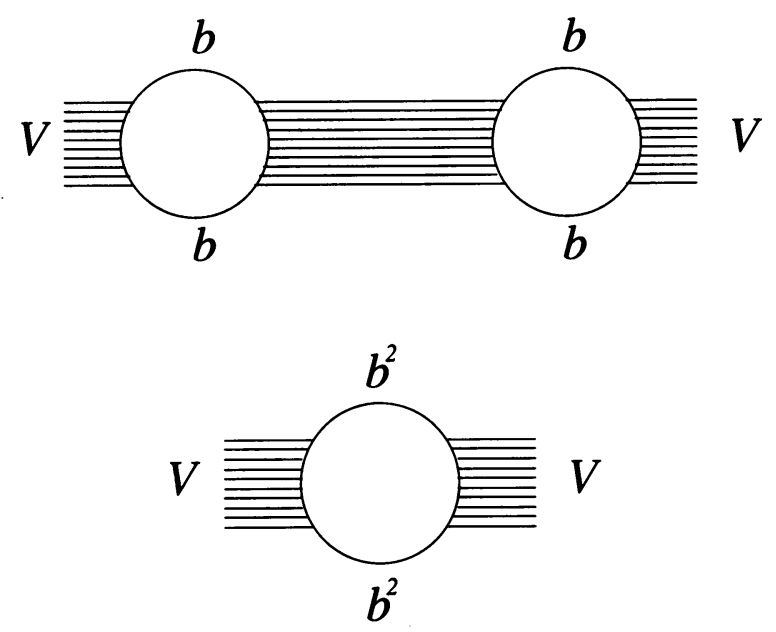

FIGURE 2

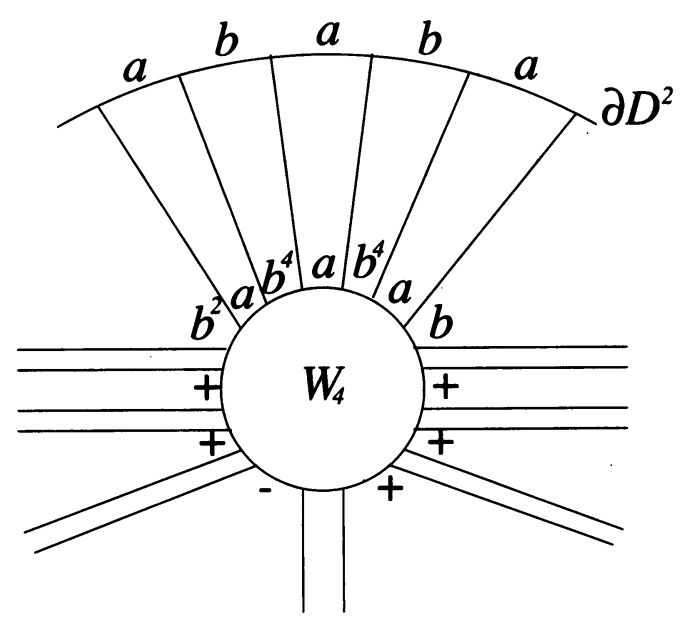

Figure 3 
Proof. The only noncyclic finite subgroups of $\operatorname{PSL}(2, \mathbb{C})$ containing elements of order 4 are $S_{4}$ and $D_{8}$, corresponding to values \pm 1 and 0 respectively for $\operatorname{tr}(A B)$. Hence $\tau_{W}$ has degree at most 3 , so that $W$ has length at most 6 , and the result follows from [12] and [11].

We are now reduced to the case where $(p, q, r)=(2,3,2)$. Conder [4] has classified all quotients of the modular group by a single relator of (free product) length at most 24. In particular this includes $W^{2}$ if $W$ has length at most 12 , so we need only consider the case where $W$ has length 14 or more.

Theorem 6.4. If $W$ is cyclically reduced of length at least 14 in $\left\langle a \mid a^{2}\right\rangle *\left\langle b \mid b^{3}\right\rangle$, then $G=\left\langle a, b \mid a^{2}, b^{3}, W^{2}\right\rangle$ is infinite, except possibly if $W$ is equivalent to one of $(a b)^{3} a b^{2} a b\left(a b^{2}\right)^{2}$, or $(a b)^{3}\left(a b^{2}\right)^{2} a b\left(a b^{2}\right)^{2}$.

Proof. Noncyclic finite subgroups of $\operatorname{PSL}(2, \mathbb{C})$ generated by an element of order 2 and an element of order 3 are $S_{3}, A_{4}, S_{4}$ and $A_{5}$. Possible traces of nontrivial elements are $0, \pm 1, \pm \sqrt{2}$ and $\frac{ \pm 1 \pm \sqrt{5}}{2}$. Hence these 9 numbers are the only possible roots for $\tau_{W}$.

Note that, if $G$ has special representations onto $S_{3}$ and onto $A_{4}$, then the induced representation onto $S_{3}^{a b} \times A_{4}^{a b} \cong \mathbb{Z}_{6}$ is also special, so that $G$ is infinite by [1]. Hence we may assume that, if 0 is a root of $\tau_{W}$, then +1 and -1 are not, and vice versa. In particular, $\tau_{W}$ has degree at most 8 , and so $W$ has length at most 16.

If $W$ has length 14 , then $\tau_{W}$ is an odd polynomial of degree 7 , so, by the above, its roots are $0, \pm \sqrt{2}$ and $\frac{ \pm 1 \pm \sqrt{5}}{2}$. In other words, $W$ must be mapped to a permutation of order 2 under each of the permutation representations: $\rho_{1}$ : $a \mapsto(12), b \mapsto(123) ; \rho_{2}: a \mapsto(12), b \mapsto(234) ;$ and $\rho_{3}: a \mapsto(12)(34), b \mapsto$ (135); but not under: $\rho_{4}: a \mapsto(12)(34), b \mapsto(123)$.

Since $G$ has no cyclic special representation, but maps to $\mathbb{Z}_{2}$ in such a way that $a$ and $W$ map nontrivially, it does not admit $\mathbb{Z}_{3}$ as a homomorphic image. It follows that the number of $b^{2}$ letters in $W$ is not congruent to 2 modulo 3. By symmetry, together with the fact that $W$ is not a proper power, we may assume that this number is either 1 or 3 . Up to equivalence, there are only four possibilities; but $\rho_{2}\left((a b)^{4}\left(a b^{2}\right)^{3}\right), \rho_{3}\left((a b)^{6} a b^{2}\right), \rho_{3}\left(a b\left(a b a b^{2}\right)^{3}\right)$ do not have order 2 , so that the only remaining case is $W=(a b)^{3} a b^{2} a b\left(a b^{2}\right)^{2}$.

If $W$ has length 16 , then $\tau_{W}$ must have roots $\pm 1, \pm \sqrt{2}$, and $\frac{ \pm 1 \pm \sqrt{5}}{2}$. Hence $\rho_{2}(W), \rho_{3}(W)$ and $\rho_{4}(W)$ must all have order 2. In particular $G$ must have $\mathbb{Z}_{3}$ as a homomorphic image, so the number of $b^{2}$ letters in $W$ must be congruent to 1 modulo 3 . By symmetry, we may assume that this number is either 1 or 4 . There are 6 possibilities up to equivalence (since $W$ is not a proper power), but none of $(a b)^{7} a b^{2},(a b)^{4}\left(a b^{2}\right)^{4},(a b)^{3} a b^{2} a b\left(a b^{2}\right)^{3}$, $(a b)^{2}\left(a b^{2}\right)^{2}\left(a b a b^{2}\right)^{2}$ are mapped to elements of order 2 by $\rho_{2}$, while $\rho_{3}\left((a b)^{2} a b^{2} a b\left(a b^{2}\right)^{2} a b a b^{2}\right)$ does not have order 2 . This reduces us to the case $W=(a b)^{3}\left(a b^{2}\right)^{2} a b\left(a b^{2}\right)^{2}$.

\section{Proof of Theorem 1.1}

Let $G=\left\langle a, b \mid a^{p}, b^{q}, W^{r}\right\rangle$ be a finite generalized triangle group that is not a triangle group (that is, $W$ is not equivalent to $a b$ ). Then $\frac{1}{p}+\frac{1}{q}+\frac{1}{r}>1$, by 
[1]. If $r>2$ then the result follows from Theorem 4.1; so assume that $r=2$. Without loss of generality we may assume that $p \leq q$. If $p>2$ then the result follows from Theorem 5.1; so assume that $p=2$. If $q=2$ then $W=a b$; so assume that $q>2$. If $q>3$ then the result follows from Lemma 6.1 and Theorems 6.2 and 6.3; so assume that $q=3$. If $W$ has length 12 or less, then the result follows from [4], while, if $W$ has length greater than 12, then the result follows from Theorem 6.4.

\section{REFERENCES}

1. G. Baumslag, J. W. Morgan and P. B. Shalen, Generalized triangle groups, Math. Proc. Cambridge Philos. Soc. 102 (1987), 25-31.

2. S. Boyer, On proper powers in free products and Dehn surgery, J. Pure Appl. Algebra 51 (1988), 217-229.

3. C. M. Campbell and R. M. Thomas, On infinite groups of Fibonacci type, Proc. Edinburgh Math. Soc. 29 (1986), 225-232.

4. M. D. E. Conder, Three-relator quotients of the modular group, Quart. J. Math. Oxford (2) 38 (1987), 427-447.

5. A. J. Duncan and J. Howie, One-relator products with high powered relations, Geometric Group Theory, vol. I (G.A. Niblo and M. Roller, eds.), London Mathematical Society Lecture Notes 181, Cambridge University Press, 1993, pp. 48-74.

6. B. Fine, J. Howie and G. Rosenberger, One-relator quotients and free products of cyclics, Proc. Amer. Math. Soc. (2) 102 (1988), 249-254.

7. B. Fine, F. Levin and G. Rosenberger, Free subgroups and decompositions of one-relator products of cyclics. Part 1: The Tits alternative, Arch. Math. 50 (1988), 97-109.

8. B. Fine and G. Rosenberger, A note on generalized triangle groups, Abh. Math. Sem. Univ. Hamburg 56 (1986), 233-244.

9. J. Howie, The quotient of a free product of groups by a single high-powered relator. I. Pictures. Fifth and higher powers, Proc. London Math. Soc. 59 (1989), 507-540.

10. J. Howie and R. M. Thomas, The groups $(2,3, p ; q)$; asphericity and a conjecture of Coxeter, J. Algebra 154 (1993), 289-309.

11. F. Levin and G. Rosenberger, On free subgroups of generalized triangle groups, Part II, Proceedings of the Ohio State-Denison Conference on Group Theory (S. Seghal et al., eds.), World Scientific, 1993, pp. 206-222.

12. G. Rosenberger, On free subgroups of generalized triangle groups, Algebra i Logika 28 (1989), 227-240.

13. R. M. Thomas, Cayley graphs and group presentations, Math. Proc. Cambridge Philos. Soc. 103 (1988), 385-387.

(J. Howie and V. Metaftsis) Department of Mathematics, Heriot-Watt University, EdINBURGH EHI 4 4AS, SCOTLAND

E-mail address, J. Howie: jim@ma.hw.ac.uk

Current address, V. Metaftsis: 107, R. Ferraiou Str., GR-383 33 Volos, Greece

E-mail address, V. Metaftsis: vasileio@ma.hw.ac.uk

(R. M. Thomas) Department of Mathematics and Computer Science, University of LEICESTER, UNIVERSITY ROAD, LEICESTER LE1 7RH, ENGLAND

E-mail address: rmt@le.ac.uk 